\title{
Metastable Pores at the Onset of Constant-Current Electroporation
}

\author{
Malgorzata Kotulska $\cdot$ Joanna Basalyga $\cdot$ \\ Maria B. Derylo $\cdot$ Przemyslaw Sadowski
}

Received: 5 January 2010/ Accepted: 7 July 2010/Published online: 23 July 2010

(C) The Author(s) 2010. This article is published with open access at Springerlink.com

\begin{abstract}
Single metastable nanopores, appearing before the actual electroporation under constant-current conditions, are used to characterize the onset of electroporation. Unlike the long-lived electropores typical of the current controlled methods, these pores survive for milliseconds and observing them is possible due to slow development of electroporation, provided by the gradual accumulation of charges on a planar membrane. Analysis of the metastable pore appearance frequency and lifetime shows the first introductory stage of electroporation. During this stage two species of metastable pores open, the majority of very low conductance that seem not fully developed as hydrophilic electropores. The experiments reveal that voltage value defines the electroporation onset while the current value affects the rate of electroporation. Membrane capacitance has a great impact on the membrane susceptibility to the pore appearance, related to its thickness and integrity. Pores of nonperfect membranes appear more easily, but they do not live any longer than others.
\end{abstract}

Keywords Electroporation - Constant current .

Metastable pore $\cdot$ Prepore

\section{Introduction}

Electroporation under constant-current conditions (Kalinowski et al. 1998) is a significantly slower process than pore formation induced by impulses of constant

M. Kotulska ( $₫)$ · J. Basalyga · M. B. Derylo · P. Sadowski Institute of Biomedical Engineering and Instrumentation,

Wroclaw University of Technology, ul. Wybrzeze

Wyspianskiego 27, 50-370 Wroclaw, Poland

e-mail: malgorzata.kotulska@pwr.wroc.pl voltage. The gentle course of such electroporation, which lasts several seconds, is due to the slow charge accumulation on the membrane, gradually leading to the conditions at which electroporation occurs. The process can be slowed down by choosing the minimum current values that may lead to electroporation. In constant-current electroporation, the appearance of the first pore lowers the transmembrane potential, which falls below the breakdown value and prevents further electroporation. Hence, in this experimental method only single pores appear, as confirmed by the total pore conductance. Controlling the current value, instead of the voltage magnitude, generates a negative feedback inherent to this method. When the fully formed electropore is expanding its radius, the membrane conductance increases, leading to reduction of transmembrane voltage. Lower values of transmembrane voltage hamper the pore expansion or even make the pore shrink. On the other hand, when the pore continues its contraction, the transmembrane potential starts rising, opposing disappearance of the pore. The constant-current method permits one to obtain a long-lived electropore of fairly good stability, which can be open to a similar extent for an hour or longer (Fig. 1). This property can be applied for studying single-pore kinetics (Kotulska et al. 2004; Kotulska 2007).

Slow evolution between the subsequent stages of electroporation extensively facilitates studying the onset of electroporation and prior phenomena. One of those early events is the formation of a metastable electropore, occurring below the typical breakdown voltage, which is unable to develop into a fully open pore capable of surviving longer than milliseconds (Fig. 2, showing the charging part of the electroporation curve from Fig. 1). We define as metastable pores the appearance of a very transient leak of ions, shown by an insignificant potential drop of single millivolts that does not last more than several 


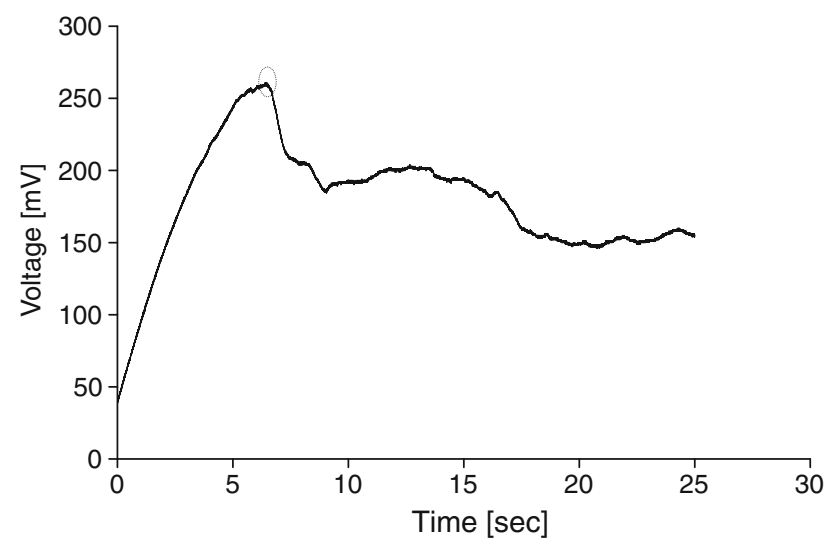

Fig. 1 Voltage course in current-clamp electroporation. Electroporation occurred after approximately $7 \mathrm{~s}$ at $250 \mathrm{mV}$, as indicated by a significant potential drop (at least several tens of $\mathrm{mV}$ ) that is not restored within less than several seconds

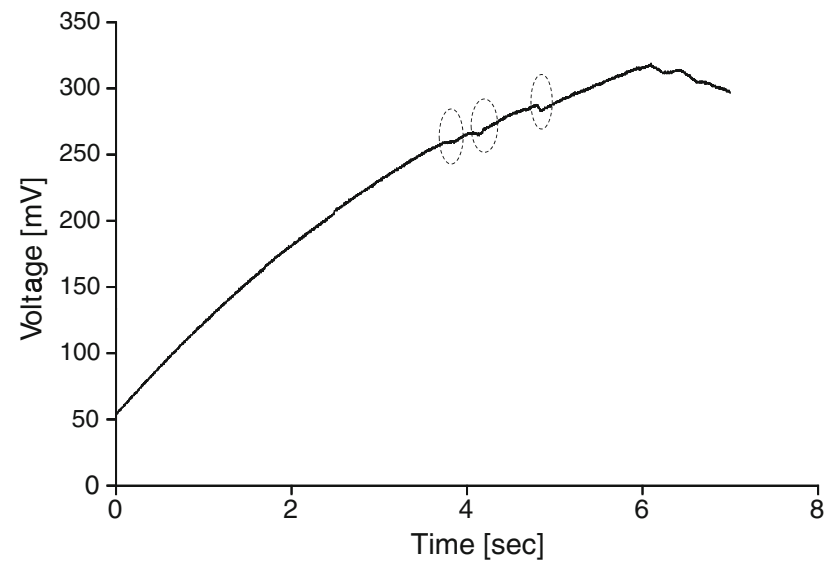

Fig. 2 The charging part of the electroporation curve here shows three metastable electropores (circles)

milliseconds, and afterward the potential is immediately restored, rising until it reaches the actual electroporation point.

The appearance of such prepores indicates limiting conditions of the actual electroporation and membrane susceptibility to the electric field, which relates to the membrane structure and mechanical properties. Our hypothesis is that metastable pores are not fully formed, exposing their hydrophobic parts to contact with microleaks of ions and water, as schematically depicted in Fig. 3. These pores tend to close when the voltage is not well above the electroporation threshold. Molecular dynamics simulations show that pore formation starts with water "wires" that invade the membrane, subsequently triggering reorganization of the lipid molecules and final formation of hydrophilic pores if the leak continues (Tieleman 2004; Tarek 2005). The hydrophilic pore, fully formed, will not reseal immediately, and even closed will keep structural changes in the membrane for some time (Melikov et al.

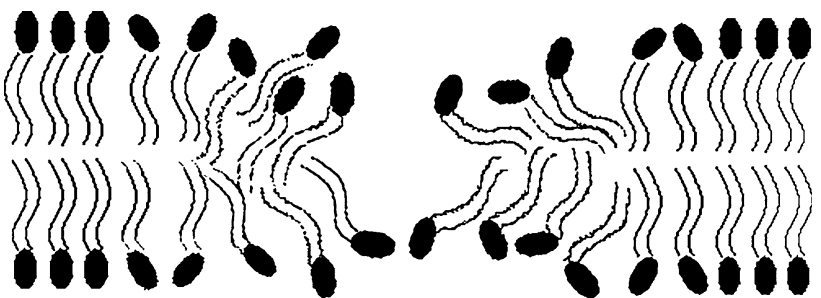

Fig. 3 Metastable pores may not be fully formed, exposing their hydrophobic parts to contact with microleaks of ions and water. Hypothetical metastable pore

2001). Resealing in the prepore experiment, presented here, should be even more difficult since the voltage is constantly rising and charge is accumulating on the membrane. The fact that metastable pores manage to close completely in such adverse conditions suggests that lipids have not fully reoriented themselves and that the pore is surrounded by abundant lipid molecules. The pores we present here could be regarded as opposite to the prepores observed by Melikov et al. (2001). In that experiment, conducted in the voltage-clamp mode, the silent and nonconductive prepores were observed at a voltage below the electroporation threshold following pulses that ensure electroporation. These prepores do not conduct ions, but they keep reoriented lipid molecules that remember the previously open hydrophilic pore. In our case the situation is reversed. The metastable pores, which also typically appear at a voltage below the electroporation threshold and can be regarded as prepores, conduct ions but the lipid molecules have not adjusted to this situation and maintain their nonreoriented position.

A study on such pores can show what is the predominant electrical quantity defining the onset of electroporation. In this work, we studied the frequency and patterns in the occurrence of metastable pores during current-clamp electroporation.

\section{Materials and Methods}

Egg yolk phosphatidylcholine (PC) was obtained from Fluka (Buchs, Switzerland), analytical-grade $\mathrm{NaCl}$ and hexahydrate $\mathrm{AlCl}_{3}$ were obtained from $\mathrm{POCH}$ (Gliwice, Poland) and n-decane was from Aldrich (Gillinghem, UK). The chemicals were used without additional purification. Electrolytes with $\mathrm{NaCl}$ were buffered with $0.01 \mathrm{M}$ HEPES (Aldrich) to $\mathrm{pH}$ 7.0. The experiments were performed on planar lipid bilayer membranes formed by the MuellerRudin method (Mueller et al. 1963) in a round aperture, $1.0 \mathrm{~mm}$ in diameter, in two Teflon hydrophobic septumseparated cells, each filled with $8 \mathrm{ml}$ of electrolyte. The experiments were performed at a temperature of about $20 \pm 1^{\circ} \mathrm{C}$. The forming solution contained PC dissolved in 
n-decane $(20 \mathrm{mg} / \mathrm{ml})$. Measurements were performed with a four-electrode $(\mathrm{Ag} / \mathrm{AgCl})$ capacitance meter and a potentiostat-galvanostat (described in Kalinowski and Figaszewski 1995), controlled by dedicated computer software. The process of membrane formation was monitored by changes in membrane capacitance. Since membrane capacitance rises when the bilayer is forming, it was assumed that the membrane was fully formed when the curve saturated and the drift $\Delta \mathrm{C}<10 \mathrm{pF} / \mathrm{min}$. The current was set at $0.18-0.25 \mathrm{nA}$, as close to the minimum current that may lead to electroporation, with a step of $0.01 \mathrm{nA}$. Tests at current values capable of inducing metastable pores were performed on 63 membranes $(10,26,10$ and 17 at $0.19,0.20,0.21$ and $0.22 \mathrm{nA}$, respectively). Membrane resistance was typically around $1 \mathrm{G} \Omega$, and pore conductance was determined as connected in parallel to the membrane conductance. Membrane capacitance, conductance and transmembrane voltage were controlled throughout the experiments with an accuracy of $2 \%$ regarding the current value.

\section{Results and Discussion}

The PC planar bilayer membrane was subjected to constant current of a value close to the lower limit capable of inducing electroporation, which exceeds the current leaks and ensures the slowest electroporation (from our experience it is above $0.15 \mathrm{nA}$ ). In several cases metastable pores were developed. We could observe them above $0.18 \mathrm{nA}$, with the maximum appearance frequency at $0.22 \mathrm{nA}$ and a decrease above this value, with no metastable pores observed at $0.24-0.25 \mathrm{nA}$ (Fig. 4). This result defines the optimal current range for studying the onset of electroporation. The experiment shows that metastable pore appearance does not depend on the current value for very low currents (the maximum at $0.22 \mathrm{nA}$ was caused by a

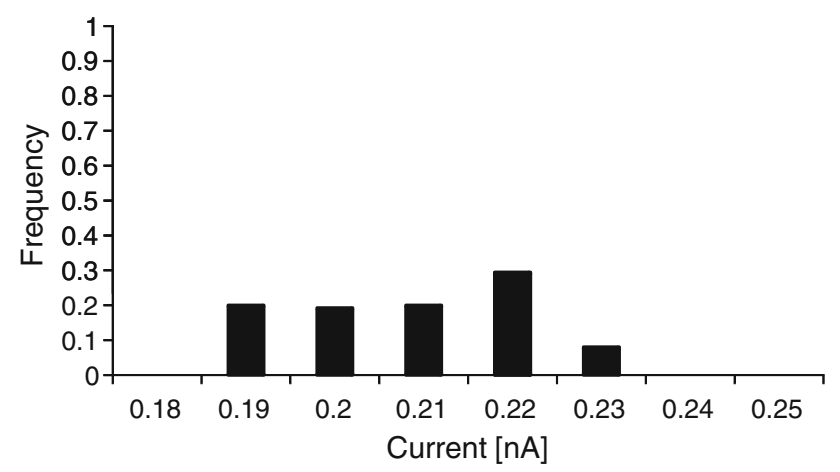

Fig. 4 Frequency of prepore appearance very weakly depends on the current value higher ratio of pores with high conductance, presented below). At higher currents, we did not observe metastable pores at our sampling frequency but the electroporation proceeded faster and the pores may have appeared and disappeared undetected.

We tested if the transmembrane voltage had more influence on the metastable pore appearance than the current value. In our experiment, these pores were observed between 130.5 and $283.8 \mathrm{mV}$. As expected, the pores most readily appeared just below the breakdown voltage, as presented in the histogram (Fig. 5), which for this setup and experimental condition is most typically around $250 \mathrm{mV}$ (Kalinowski et al. 1998; Kotulska et al. 2004; Kotulska 2007). The value of the electroporation threshold is specific for the membrane composition, dimensions and shape. It is different for cells and planar lipid membranes, but it also differs between various experimental methods applied to similar planar bilayers. For example, the experiment with linearly rising voltage showed a decreased electroporation threshold for slowly rising potential (Kramar et al. 2007), which accounts for the slightly lower electroporation values in the current-clamp methods and voltage pulses.

Metastable pores were not observed under a transmembrane potential of $130 \mathrm{mV}$. The pore appearance very clearly depends on the transmembrane voltage, with the peak just below the breaking potential. Most metastable pores detected above $210 \mathrm{mV}$ showed larger conductance, $>60 \mathrm{pS}$. For voltages $>270 \mathrm{mV}$, no prepores were observed; but in this limit full electroporation is very likely to take place, in which a proper long-lived hydrophilic pore forms.

The observed conductance of the metastable pores fell into the range 3.2-79.5 pS, although the low-conductance pores were predominant and vividly separated from the higher-conductance pores, usually appearing just before the actual electroporation, as presented in the histogram

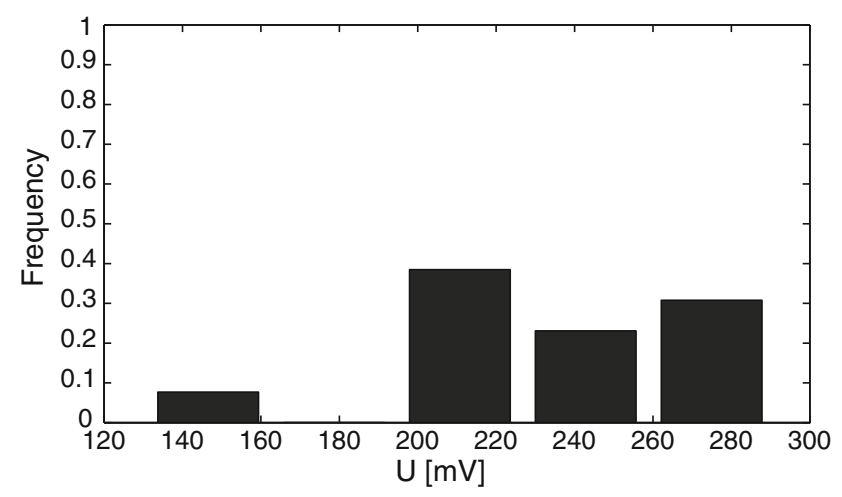

Fig. 5 Frequency of prepore appearance depends on transmembrane voltage, and it is highest just below the electroporation potential value (histogram) 


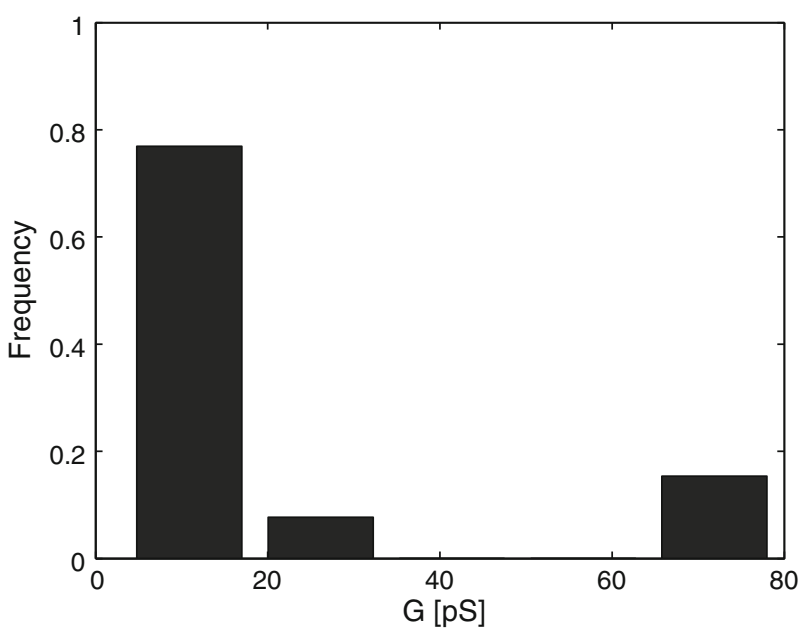

Fig. 6 Metastable pores with low conductance are predominant and clearly separated from the higher-conductance pores, usually appearing close to full electroporation (histogram)

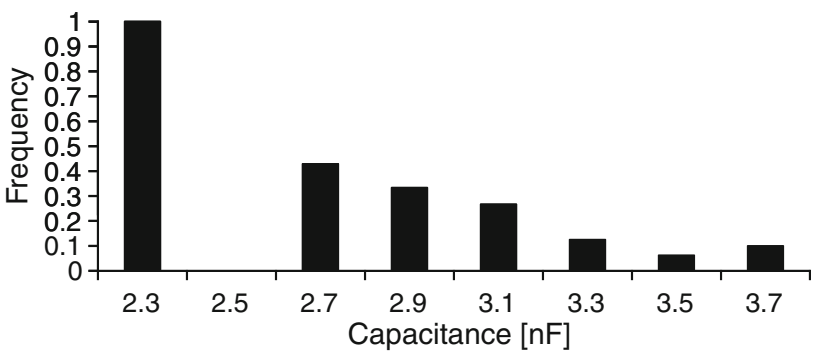

Fig. 7 Frequency of prepore appearance depends on membrane capacitance

(Fig. 6). The majority of these high-conductance pores appeared at a current of $0.22 \mathrm{nA}$, generating the frequency maximum in Fig. 4.

In experiments where metastable pores were observed, the mean specific capacitance per unit area of the membranes was $0.4 \pm 0.06 \mu \mathrm{F} / \mathrm{cm}^{2}$. Analysis of the influence of capacitance on the appearance of metastable pores showed a dependence between appearance frequency and membrane capacitance (Fig. 7, the capacitance values are represented by central values of the intervals). Metastable pores appear on the membranes with low capacitance more easily, which may be related to the membrane thickness. In this type of experiment, the membrane forms by gradual reorganization of the lipid molecules - chunks of randomly oriented lipids form a classical thin bilayer without solvent molecules inside the membrane. A thicker bilayer of lower conductance may mean that not all molecules managed to orient themselves properly. Such a membrane can be full of defects, weakening intermolecular interactions and membrane integrity. This can result in lower endurance to the electric field and finally a higher chance of metastable pore appearance.

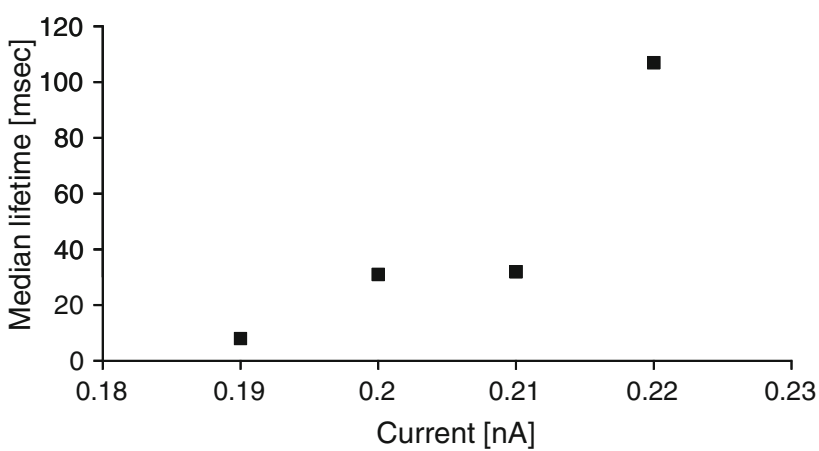

Fig. 8 Median lifetimes of metastable pores depend on the current value

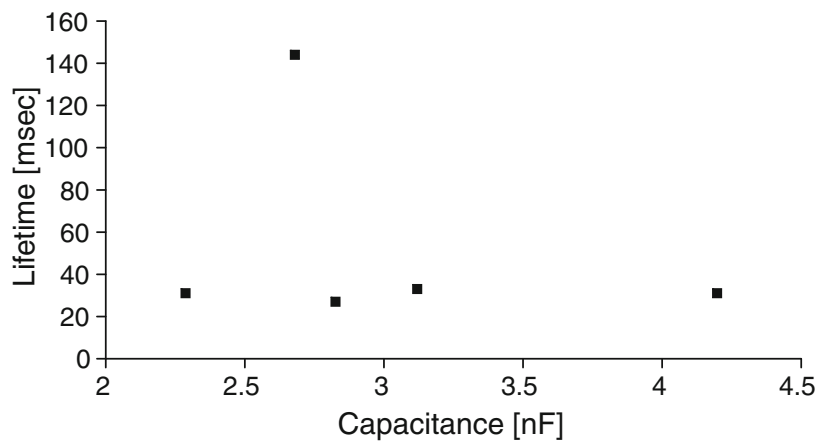

Fig. 9 Lifetime of the prepore is independent of membrane capacitance (data for $I=0.2 \mathrm{nA}$ )

While a metastable pore was formed, we studied its median lifetime. Although the current value had little impact on the appearance frequency, the pore lifetime depends on the current value, and higher currents prevent fast disappearance of the pore (Fig. 8). This can be explained by increased chance of proper, long-lived pore formation for greater current values.

A similar study, however involving membrane capacitance, showed no dependence of the pore lifetime on membrane capacitance (Fig. 9 shows nonaveraged data for $I=0.22 \mathrm{nA}$ ). This may seem slightly surprising when the appearance frequency is recalled (Fig. 7), but it shows that pores opening in nonperfect membranes only appear more easily but do not tend to behave any more similar to the proper long-lived electropores. We did not observe any relation between pore lifetime and its conductance (data not shown).

\section{Conclusions}

Metastable electropores, which appear at the onset of the current-clamp experiment, show the first stage of membrane electroporation. Their observation is possible due to 
slow development of the process in such experiments, provided by gradual accumulation of the charges on the membrane at low current values. The study showed that transmembrane potential value is more relevant in triggering the event of electroporation than the current value. The current value, however, defines the speed of the membrane charging and pace of the transitions between subsequent stages of electroporation. Therefore, the current level affects the lifetime of the transient pores. Although all of the studied metapores were not stable and typically survived up to tens of milliseconds, they were not uniform. Two distinct species were observed in our experiments. Most of them were very small pores of conductance $<15 \mathrm{pS}$, and a few pores were larger. Although the first set can consist of pores that are not fully developed, the latter category is obviously more related to classical long-lived electropores appearing in current clamp experiments, which live from $10 \mathrm{~s}$ to several hours, typically causing a voltage drop of $100-150 \mathrm{mV}$. The study showed that the planar membrane is significantly more susceptible to electroporation when its capacitance is low, which is probably related to its thickness and structure. However, membranes with low capacitance, although less resistant to electric field, are not more likely to develop pores of longer lifetime. This may indicate that early pores in such membranes can also resemble short-lived defects rather than proper electropores. Our study showed the main features of metastable pores, which gave more insight into the process of electropore development and the relationships between their appearance and conditions of the lipid bilayer.

Acknowledgements We thank Prof. Damijan Miklavcic for bringing the idea of metastable pores to our attention. P. S., J. B. and
M. B. D. were supported by the grant for students association Bio-Nanopore from Wroclaw University of Technology.

Open Access This article is distributed under the terms of the Creative Commons Attribution Noncommercial License which permits any noncommercial use, distribution, and reproduction in any medium, provided the original author(s) and source are credited.

\section{References}

Kalinowski S, Figaszewski Z (1995) A four-electrode system for measurements of bilayer lipid membrane capacitance. Meas Sci Technol 6:1043-1049

Kalinowski S, Ibron G, Bryl K, Figaszewski Z (1998) Chronopotentiometric studies of electroporation of bilayer lipid membranes. Biochim Biophys Acta 1369:204-212

Kotulska M (2007) Natural fluctuations of an electropore show fractional Lévy stable motion. Biophys J 92:2412-2421

Kotulska M, Koronkiewicz S, Kalinowski S (2004) Self-similar processes and flicker noise from a fluctuating nanopore in a lipid membrane. Phys Rev E 69:031920-031930

Kramar P, Miklavcic D, Lebar AM (2007) Determination of the lipid bilayer breakdown voltage by means of linear rising signal. Bioelectrochemistry 70:23-27

Melikov KC, Frolov VA, Shcherbakov A, Samsonov AV, Chizmadzhev YA, Chernomordik LV (2001) Voltage-induced nonconductive prepores and metastable single pores in unmodified planar lipid bilayer. Biophys J 80:1829-1836

Mueller P, Rudin DO, Tien HT, Wescott WC (1963) Methods for the formation of single bimolecular lipid membranes in aqueous solutions. J Phys Chem 67:534-535

Tarek M (2005) Membrane electroporation: a molecular dynamics simulation. Biophys J 88:4045-4053

Tieleman DP (2004) The molecular basis of electroporation. BMC Biochem 5:10 